\title{
Bewegungsstörungen - wenig beachtete Aspekte von großer praktischer Relevanz
}

D ie Bewegungsstörungen sind mit diesem Heft erneut Schwerpunkt von psychoneuro. Das Augenmerk wird dabei insbesondere auf Aspekte gesetzt, die trotz großer Relevanz für die betroffenen Patienten sowohl in der wissenschaftlichen Literatur als auch häufig bei der ärztlichen Betreuung nicht ausreichend beachtet werden. Dies gilt insbesondere für die Depression bei Parkinson-Patienten, die fast die Hälfte aller Parkinson-Patienten betrifft, wie M.R. Lemke in seiner umfassenden Übersicht aufzeigt. Obwohl klinische und experimentelle Untersuchungen auf eine anti-depressive Wirkung der neueren Dopaminagonisten über kortiko-frontale $\mathrm{D}_{3}$-Rezeptoren hinweisen, mangelt es leider nahezu komplett an kontrollierten Studien zur Depressionsbehandlung beim M. Parkinson.

Eine weitere Übersichtsarbeit dieses Heftes beschäftigt sich mit dem geriatrischen Parkinson-Patienten. Insbesondere werden die pharmakokinetischen und pharmakodynamischen Besonderheiten der Anti-Parkinson-Therapie im hohen Lebensalter aufgezeigt.

Das Restless-legs-Syndrom gehört zu den häufigsten neurologischen Erkrankungen überhaupt. Zirka 10\% der über Fünfundsechzigjährigen sind davon betroffen, wie epidemiologische Untersuchungen aus dem Raum Nürnberg und aktuell aus Vorpommern zeigen. Unverständlicherweise wird es jedoch weiterhin nicht erkannt. H. Beneš hat intensiv insbesondere zur Pharmakotherapie des RLS gearbeitet. Sie gibt unter dem Titel „Was gibt es Neues zum Restless-legs-Syndrom?" eine praktisch orientierte Übersicht zu diesem Krankheitsbild, das auch bereits Kinder betreffen kann.
H. Topka fasst den Bereich der hyperkinetischen Bewegungsstörungen zusammen. Wichtig ist es insbesondere auch, einzelne Sonderformen, wie die L-Dopa-responsive Dystonie oder paroxysmale Dystonien zu erkennen, da hier eine sehr erfolgreiche Pharmakotherapie möglich ist.

Den Autoren gilt mein Dank für die rasche Zusage, ein Manuskript für dieses Heft von psychoneuro zu erstellen, und die termingerechte Fertigstellung ihrer interessanten, da praxisorientierten Beiträge. Den Lesern wünsche ich viele nützliche Hinweise für die praktische Arbeit.

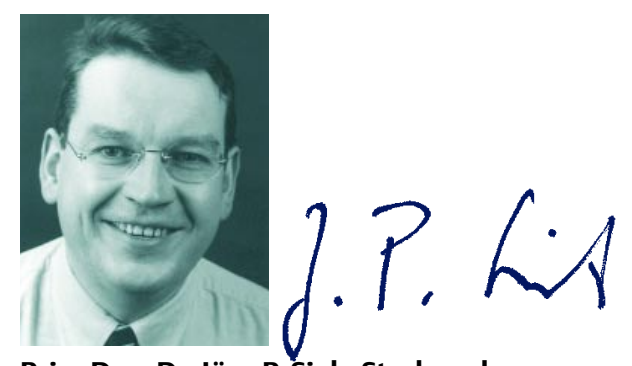

Priv.-Doz. Dr. Jörn P. Sieb, Stralsund 